\title{
Design and Self-Consistent Schrodinger-Poisson Model Simulation of Ultra-Thin Si-Channel Nanowire FET
}

\section{Chhaya Verma}

NIT Sikkim: National Institute of Technology Sikkim

Jeetendra Singh ( $\square$ jeetendra@nitsikkim.ac.in )

National Institute of Technology Sikkim

\section{Research Article}

Keywords: Nanowire FET, quantum mechanical models, non-equilibrium green function, Threshold voltage, Sub-threshold swing

Posted Date: August 13th, 2021

DOI: https://doi.org/10.21203/rs.3.rs-795778/v1

License: (c) (1) This work is licensed under a Creative Commons Attribution 4.0 International License.

Read Full License 


\title{
Design and Self-Consistent Schrodinger-Poisson model
}

\section{Simulation of Ultra-Thin Si-Channel Nanowire FET}

\author{
Chhaya Verma ${ }^{1}$, Jeetendra Singh ${ }^{2 *}$ \\ ${ }^{1,2}$ Department of ECE, National Institute of Technology Sikkim, India \\ *jeetendra@nitsikkim.ac.in
}

Abstract: Since at the regime of nanometer, the quantum confinement effects are observed and the wave nature of electrons is more dominant. Therefore, the classical approach of current formulation in mesoelectonics and nanoelectronics results in inaccuracy as it does not consider the quantum effect, which is only applicable for the bulk electronic device. For accurate modeling and simulation of nanoelectronics, device atomic-level quantum mechanical models are required. In this work, an ultra-thin (3 nm diameter) Silicon- channel Cylindrical Nanowire FET (CNWFET) is designed and simulated by invoking non-equilibrium green function (NEGF) formalism and self-consistent Schrodinger-Poisson's equation model. Then impact variation of temperature, oxide thickness, and metal work function variation in the proposed NWFET is investigated to analyze the distinct performance parameters of the device e.g. threshold voltage (Vth) drain induced barrier lowering (DIBL), sub-threshold swing (SS), and $\mathrm{I}_{\mathrm{ON}} / \mathrm{I}_{\mathrm{OFF}}$ ratio. The designed device exhibits reliable results and shows a SS of $57.8 \mathrm{mV} /$ decade and Ion to IOFF ratio of order $10^{9}$ at room temperature.

Keywords: Nanowire FET, quantum mechanical models, non-equilibrium green function, Threshold voltage, Sub-threshold swing

\section{Introduction}

The demand to scale down MOSFET to increase the chip density with high performance has forced the researcher to look for another device as MOSFET has reached its physical limit [1]. In 2010, Cheung et al. stated that the subthreshold swing of MOSFETs can never be below $60 \mathrm{mV} / \mathrm{decade}$ at room temperature [2]. The further scaling of the MOSFET channel roughly below $65 \mathrm{~nm}$ begins to exhibit 
quantum phenomena shows high leakage source to drain OFF current and control of gate is lost over the device [3-5]. Moreover, the limitation of the sub-threshold swing will lead to high leakage current even in the OFF state resulting in static power dissipation which finally may result in the thermal runway of the electronic device $[6,7]$. To maintain pace with Moore's Law and pack more and more transistors together, the researcher has been working beyond conventional semiconductor technology [8]. Since the main aim is a small-sized transistor with high performance and low power consumption, many changes in MOSFET structure and MOSFET-like structure were done and but with persistent short channel effects, the parasitic capacitance was observed and very little improvement in the subthreshold swing was achieved [9-11]. In case if the device succeeded in low SS then the ON/OFF current ratio was compromised. The novel Nanoscaled semiconductor devices like nanotube TFETs, Nanowire FET and CNT, etc. are very promising for future electronic systems [12-14]. But the study and simulation of the nanoscaled devices are challenging as at nanoscale regime, the transistor operates near the ballistic limit, electron-hole pairs are generated by the band to band tunneling despite thermal emission and show quantum effects $[15,16]$.

The classical models of the simulation are optimal for the bulk device but for the mesoscopic and nanoscopic electronics devices, quantum mechanical models are required. Therefore, to model quantum confinement effects, the Self- consistent Schrodinger-Poisson model which uses the 1D NEGF method for computation of current is invoked and ultra-thin silicon channel cylindrical NWFET is designed by considering the nanowires to be a 1-D nanostructure. Then, the impact of work function variation, oxide thickness variation, and temperature variation are investigated to analyze device performance.

\section{Device Design and Simulation Model}

The 3D-designed structure of cylindrical nanowire FET (CNWFET) is shown in Fig. 1(a). The diameter of the cross-section is chosen as $3 \mathrm{~nm}\left(2 \mathrm{~nm} \mathrm{Si}+1 \mathrm{~nm} \mathrm{SiO}_{2}\right)$. The shown Fig. 1(b) is of the twodimensional structure of the designed CNWFET which has been used for current simulation and is 
derived from cutting the three-dimensional structure along the Y-Z plane. The vertical length of the proposed NWFET is $50 \mathrm{~nm}$ with a channel length of $30 \mathrm{~nm}$ and drain and source region length is $10 \mathrm{~nm}$ each. The source and drain are doped with an N-type uniform doping profile of $1 \times 10^{18} / \mathrm{cm}^{3}$ and $1 \times 10^{20} / \mathrm{cm}^{3}$ whereas the channel is doped with a P-type doping profile of $1 \times 10^{15} / \mathrm{cm}^{3}$. The various design parameters of the device are mentioned in Table 1.
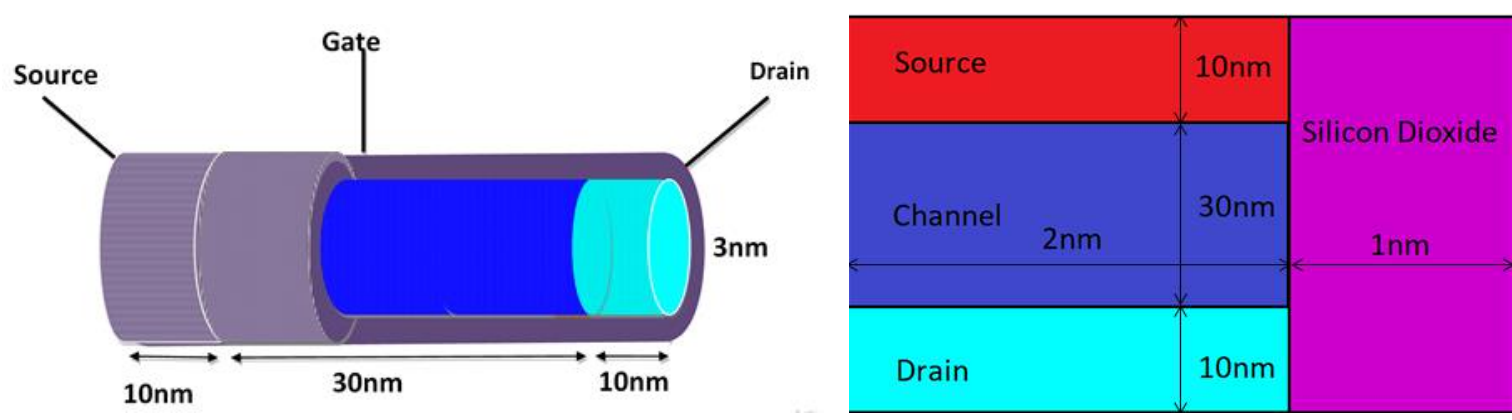

Fig. 1(a) Three-dimensional structure of proposed Cylindrical Nanowire FET (CNWFET) (b) Twodimensional structure of proposed Cylindrical Nanowire FET (CNWFET)

Table I: various design parameters of proposed cylindrical NWFET

\begin{tabular}{|l|l|l|}
\hline S. No. & Design Parameters & Values \\
\hline 1 & Channel length & $30 \mathrm{~nm}$ \\
\hline 2 & Gate work function & $4.7 \mathrm{eV}$ \\
\hline 3 & $\mathrm{SiO}_{2}$ thickness & $1 \mathrm{~nm}$ \\
\hline 4 & Si layer thickness & $2 \mathrm{~nm}$ \\
\hline 6 & Source doping Concentration & n-type $1 \times 10^{15} / \mathrm{cm}^{3}$ \\
\hline 7 & & \\
\hline 8 & Drain doping Concentration & n-type $1 \times 10^{20} / \mathrm{cm}^{3}$ \\
\hline & & \\
\hline
\end{tabular}


Since the diameter of the designed device is $3 \mathrm{~nm}$, it requires quantum mechanical models for its accurate simulation. The Self-consistent coupled Schrödinger Poisson model uses Schrodinger relation to obtain the density of state and Poisson equation to derive the potential which is further used in solving Schrödinger equations. The one-dimensional quantum confinement (say Y-axis), 1D Schrodinger equation (1) is used.

$$
-\frac{\eta}{2} \frac{\partial}{\partial x}\left(\frac{1}{m_{y}^{v}(x, y)} \frac{\partial \psi_{i v}}{\partial x}\right)+E_{C}(x, y) \psi_{i v}=E_{i v} \psi_{i v}
$$

The equation (1) is solved to get Eigen state energies $E_{i v}(x)$ and wave function $\psi_{i v}(x, y)$ at each node perpendicular to $\mathrm{X}$-axis. Here, $\mathrm{E}_{\mathrm{C}}(\mathrm{x}, \mathrm{y})$ is the band edge energy. The $m_{y}^{v}(x, y)$ represents effective mass in the $\mathrm{Y}$ direction and it should be noted these effective mass are dimension dependent and varies with the direction. The general Poisson equation is given by

$$
\nabla(\varepsilon \nabla V)=-\rho
$$

Where $\varepsilon$ is material-dependent permittivity, $\mathrm{V}$ is the electrostatic potential, and $\rho$ is the charge density. The density of state obtained by solving equation (1) are discrete and are reduced to a sum over bound state energies from integral over energy by applying Fermi-Dirac statistics. Thus the carrier concentrations obtained for $1 \mathrm{D}$ confinement is given as

$$
N(x, y)=2 \frac{k_{B} T}{\pi \eta^{2}} \sum_{v} \sqrt{m_{x}^{v}(x, y) m_{z}^{v}(x, y)} \sum_{i=0}^{\infty}\left|\psi_{i v}(x, y)\right|^{2} \ln \left[1+\exp \left(-\frac{E_{i v}-E_{F}}{K_{B} T}\right)\right]
$$

The evaluated carrier concentrations from equation (3) are substituted in net charge density in Poisson's relation. Since the net charge density is given by 


$$
\rho=q\left[N_{\text {Doping }}-N(x, y)\right]
$$

Where $q$ is the absolute electron charge, $\mathrm{N}_{\text {Doping }}$ is doping atom concentration in the Silicon film and $\mathrm{N}_{(\mathrm{x}, \mathrm{y})}$ is the calculated electron density. Thus, the potential derived from solving the Poisson equation (3) is substituted back into Schrodinger's equation. This is an iterative process between Schrodinger's and Poisson's equation which continues until convergence and a self-consistent solution of Schrodinger's and Poisson's equation is obtained. A predictor-corrector scheme using the non-equilibrium green function (NEGF) model is used for the stable and oscillation-less iteration process. After multiple iterations, wave functions are obtained and remain constant, where Eigen energies are constantly revised with each iteration and substituted in Poisson's equation. In this scheme, the Poisson equation act as a predictor, and the number of predictor iteration is controlled by the NEGF model.

$$
J_{n}=-q \mu_{n} N \nabla \phi+q D_{n} \nabla N
$$

The final solution of this self-consistent coupled Schrodinger-Poisson equation is incorporated in the drift-diffusion model (DDM) to obtain the current density and final drain current of the device. Equation (5) is a general drift-diffusion equation. The designed device is simulated in the atlas silvaco tool and during simulation drift-diffusion space model (dd_ms) along with Schrodinger (p.schro) and (ox. schro) are utilized to consider floating body effects and quantum confinements in cylindrical NWFET. Generation-recombination phenomena like Shockley-Read-Hall (srh), impact ionization, band to band tunneling and Auger models are also here.

\section{Results and Discussions}

To study the effect of various device design parameters on the performance of the device, drain current, electric field, Potential, are plotted through simulation. To analyze the impact of device design parameters 
on the performance of the device, the parameters such as silicon dioxide thickness (Tox), temperature, and gate work function are also varied.

\section{A. Transfer and Output Characteristics}

The transfer characteristics $\left(\mathrm{I}_{\mathrm{D}}-\mathrm{V}_{\mathrm{GS}}\right)$ of the proposed cylindrical NWFET device including the design parameter mentioned in Table 1 are plotted in Fig. 2 for $\mathrm{V}_{\mathrm{DS}}=1 \mathrm{~V}$. The $\mathrm{ON}$-current $\left(\mathrm{I}_{\mathrm{ON}}\right)$ at $\mathrm{V}_{\mathrm{GS}}=1 \mathrm{~V}$ and $\mathrm{V}_{\mathrm{GS}}=1.5 \mathrm{~V}$ is noticed as $6.70 \times 10^{-7}(\mathrm{~A} / \mu \mathrm{m})$ and $8.67 \times 10^{-7}(\mathrm{~A} / \mu \mathrm{m})$ whereas the OFF-current $\left(\mathrm{I}_{\mathrm{OFF}}\right)$ is observed as $5.67 \times 10^{-16}(\mathrm{~A} / \mu \mathrm{m})$ and leads the $\mathrm{I}_{\mathrm{ON}} / \mathrm{I}_{\mathrm{OFF}}$ ratio in the range of $10^{9}$. Fig. 3 shows the output characteristics $\left(I_{D}-V_{D S}\right)$ of the proposed device for $V_{G S}=0.6 V$ and inset shows $\left(I_{D}-V_{D S}\right)$ variation for $I_{D^{-}}$ $\mathrm{V}_{\mathrm{GS}}=0 \mathrm{~V}$, drain current varies from $2.57 \times 10^{-10}(\mathrm{~A} / \mu \mathrm{m})$ to $1.13 \times 10^{-7}(\mathrm{~A} / \mu \mathrm{m})$ for $\mathrm{V}_{\mathrm{GS}}=0.6 \mathrm{~V}$ and it varies between $1.13 \times 10^{-14}(\mathrm{~A} / \mu \mathrm{m})$ to $2.8 \times 10^{-16}(\mathrm{~A} / \mu \mathrm{m})$ for $\mathrm{V}_{\mathrm{GS}}=0 \mathrm{~V}$.

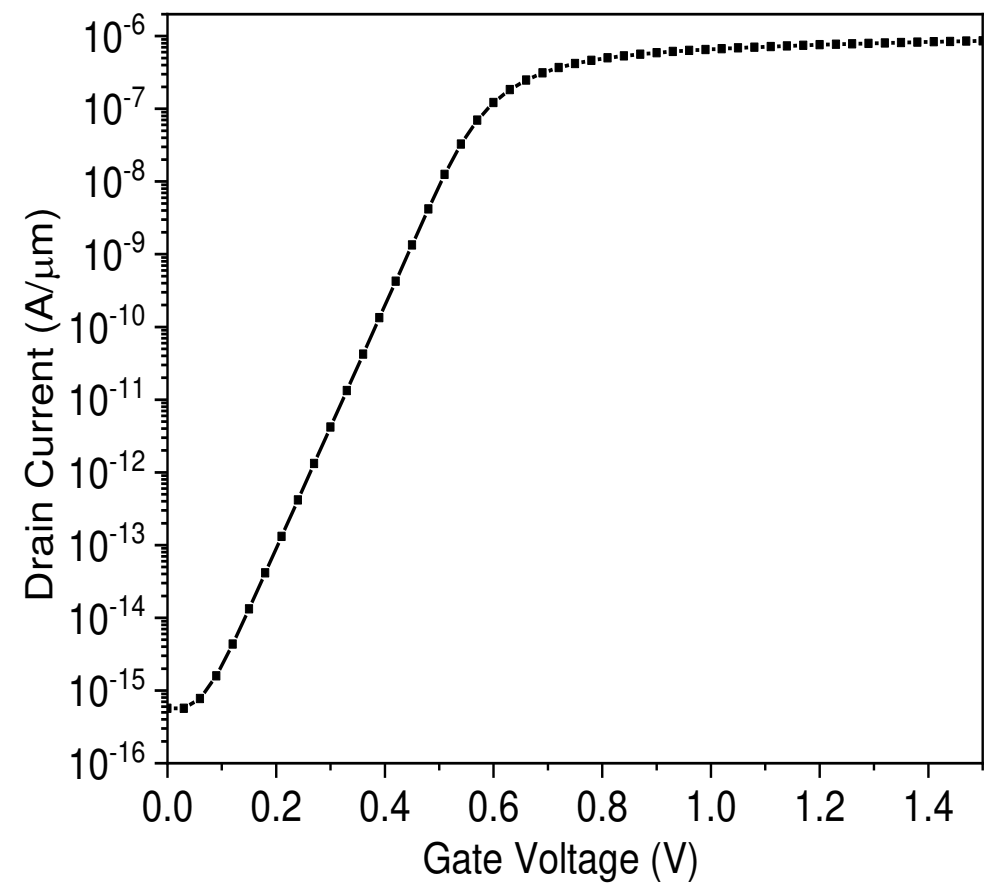

Fig. 2 the transfer characteristics $\left(I_{D}-V_{G S}\right)$ of the proposed cylindrical NWFET device for $V_{D S}=1 V$ 


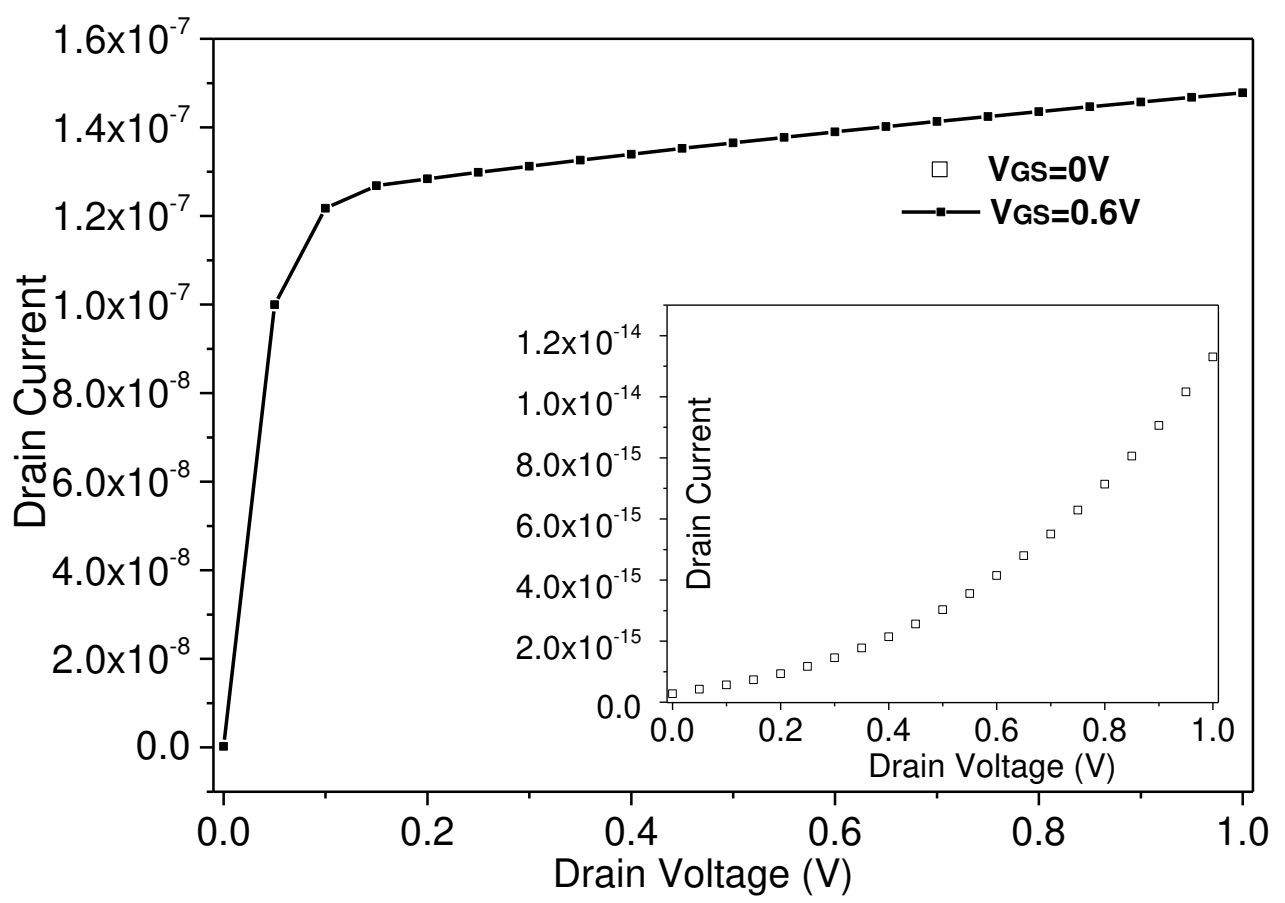

Fig. $3 \mathrm{I}_{\mathrm{D}}-\mathrm{V}_{\mathrm{DS}}$ Characteristics of proposed NWFET at $\mathrm{V}_{\mathrm{GS}}=0.6 \mathrm{~V}$ and $\mathrm{V}_{\mathrm{GS}}=0 \mathrm{~V}$

\section{B. Impact of Temperature Variation}

To study the effect of temperature on the designed nanowire FET, the device is simulated at five different temperatures which are $273 \mathrm{~K}, 300 \mathrm{~K}, 310 \mathrm{~K}, 323 \mathrm{~K}$, and $373 \mathrm{~K}$. The obtained $\left(\mathrm{I}_{\mathrm{D}}-\mathrm{V}_{\mathrm{GS}}\right)$ characteristics, electric field, and potential variation at distinct temperatures are shown in Fig. 4. The uniform doping of source, drain, and the channel is maintained constant at $1 \times 10^{15} / \mathrm{cm}^{3} \mathrm{n}$-type, $1 \times 10^{20} / \mathrm{cm}^{3} \mathrm{n}$-type, and $1 \times 10^{15} / \mathrm{cm}^{3}$ p-type respectively. The effect temperature variation is highly observed on IoFf as with increasing temperature the $\mathrm{OFF}$ current increases but the $\mathrm{I}_{\mathrm{ON}}$ is very less affected by temperature variation. In Fig. 4(b), it is observed that the temperature effect on the electric field is minimal and the potential curve tends to shift downward with increasing temperature as shown in Fig. 4(c). The subthreshold swing is $54.4 \mathrm{mV} / \mathrm{dec}$ at $273 \mathrm{~K}$ temperature and on increasing the temperature the SS increases which justifies the fact that sub-threshold swing is a function of temperature [2], $\mathrm{SS}(\mathrm{T}) \approx \ln 10 \frac{K T}{q}$. The variations of the various performance parameters like threshold voltage $\left(\mathrm{V}_{\text {th }}\right)$, drain induced barrier lowering (DIBL), SS, and $\mathrm{I}_{\mathrm{ON}} / \mathrm{I}_{\mathrm{OFF}}$ ratio with temperatures are shown in Table-II; these 
values are evaluated at the $\mathrm{SiO}_{2}$ thickness of $1 \mathrm{~nm}$, and gate work function of $4.7 \mathrm{eV}$. It can be seen from Table II that the threshold voltage and $\mathrm{I}_{\mathrm{ON}} / \mathrm{I}_{\mathrm{OFF}}$ ratio decreases with the temperature whereas DIBL and SS increase with the temperature.
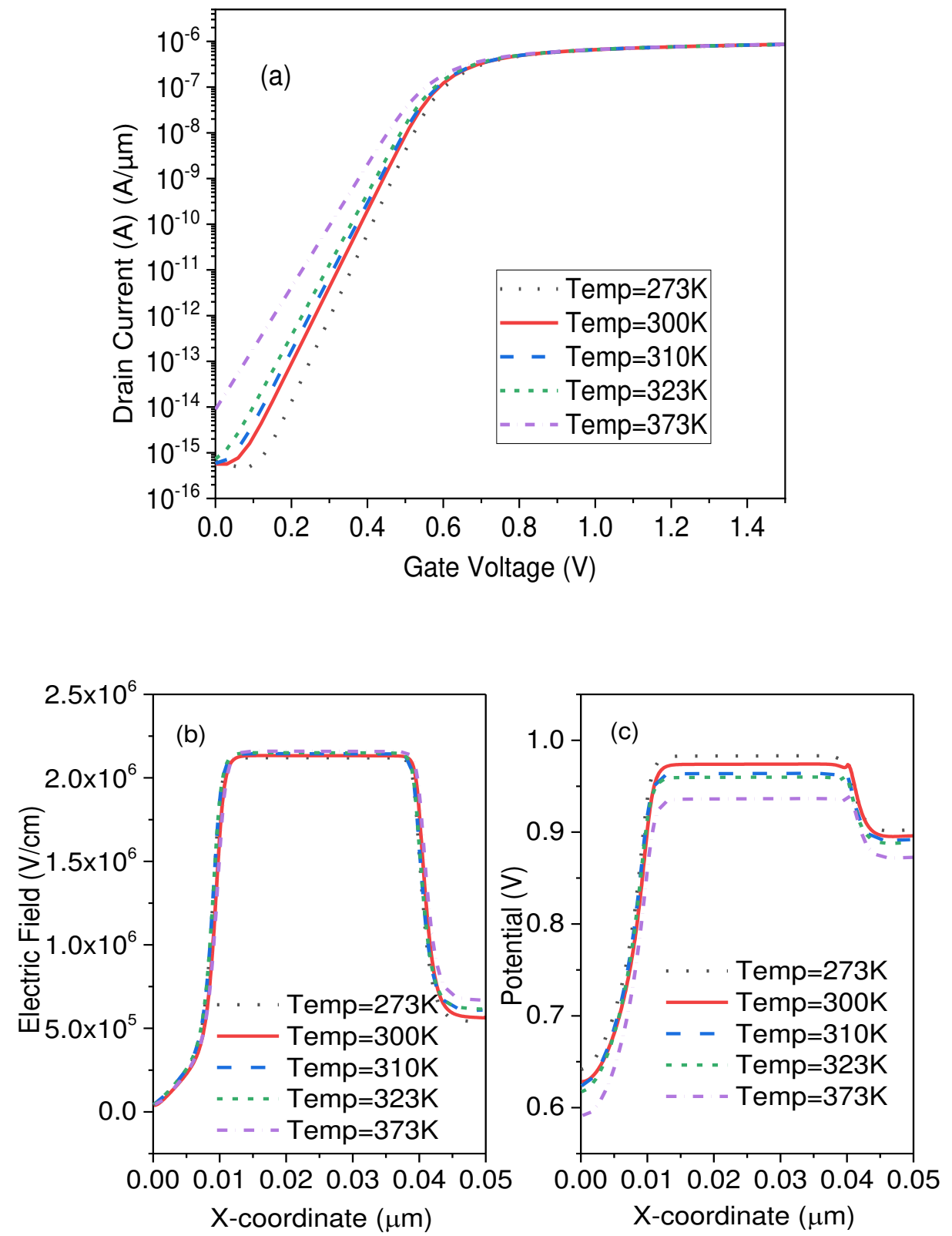

Fig. 4 (a) $\mathrm{I}_{\mathrm{D}}-\mathrm{V}_{\mathrm{GS}}$ characteristics of the proposed NWFET for variable temperature, Fig. 4 (b): Impact of variable temperature on Electric Field(V/cm), Fig. 4(c) Impact of variable temperature on the potential (V) 
Table II: Variation of various performance parameters for different temperature

\begin{tabular}{|l|l|l|l|l|l|}
\hline SI.No. & Temperature(K) & Threshold & DIBL(V/V) & $\begin{array}{l}\text { Sub-threshold } \\
\text { Swing(mV/dec) }\end{array}$ & Ion/loff Ratio \\
\hline 1 & 273 & Voltage (V) & & 54.3947 & $1.00702 \times 10^{9}$ \\
\hline 2 & 300 & 0.589483 & 5.57474 & 57.80 & $1.16112 \times 10^{9}$ \\
\hline 3 & 310 & 0.584566 & 5.76593 & 61.8041 & $1.12349 \times 10^{9}$ \\
\hline 4 & 323 & 0.577842 & 6.02453 & 64.4098 & $9.07174 \times 10^{8}$ \\
\hline 5 & 373 & 0.55377 & 7.057 & 74.443 & $7.46655 \times 10^{7}$ \\
\hline
\end{tabular}

\section{Silicon-Oxide (SiO2) Thickness Variation}

To examine the impact of $\mathrm{SiO}_{2}$ thickness variation on the device performance, the silicon oxide thickness is varied from $0.5 \mathrm{~nm}$ to $1 \mathrm{~nm}$ at constant room temperature and the corresponding $\mathrm{I}_{D}-\mathrm{V}_{\mathrm{GS}}$ characteristics, electric field variation, and potential variations are plotted in Fig. 5. It can be observed from the $\mathrm{I}_{\mathrm{D}}-\mathrm{V}_{\mathrm{GS}}$ ) characteristics are shown in Fig. 5 (a) that decreasing the oxide thickness increases both the ION and IOFF but the increase is minimal. The doping concentration of source, drain, and channel regions are maintained constant at $1 \times 10^{15} / \mathrm{cm}^{3}$ n-type, $1 \times 10^{20} / \mathrm{cm}^{3}$ n-type, and $1 \times 10^{15} / \mathrm{cm}^{3}$ p-type respectively. The effect of $\mathrm{SiO}_{2}$ thickness variation on device parameters such as potential and electric field is shown in Fig. 5 (a) and 5(b) which show augmentation in both electric field and surface potential of the device. The variations of the other performance parameters; $\mathrm{V}_{\mathrm{th}}$, DIBL, SS, and $\mathrm{I}_{\mathrm{ON}} / \mathrm{I}_{\mathrm{OFF}}$ ratio for various oxide thickness are summarized in Table-III, these values are evaluated at the gate work function of $4.5 \mathrm{eV}$ and it is seen that $\mathrm{I}_{\mathrm{ON}} / \mathrm{I}_{\mathrm{OFF}}$ ratio is the least for $1 \mathrm{~nm} \mathrm{SiO} 2$ thickness. And it is also noticed that the impact of $\mathrm{SiO}_{2}$ thickness variation is negligible on the subthreshold swing but DIBL is significantly varying with varying $\mathrm{SiO}_{2}$ thickness. 
Table III: Variation of various performance parameters for different $\mathrm{SiO}_{2}$ thickness

\begin{tabular}{|c|c|c|c|c|c|}
\hline $\begin{array}{l}\text { SI.N } \\
\text { o. }\end{array}$ & $\begin{array}{l}\text { Silicon-Oxide } \\
\text { thickness }\end{array}$ & $\begin{array}{l}\text { Threshold } \\
\text { Voltage (V) }\end{array}$ & DIBL (V/V) & $\begin{array}{l}\text { Sub-threshold } \\
\text { Swing(mV/dec) }\end{array}$ & Ion/IOFF Ratio \\
\hline 1 & Tox $=0.5 \mathrm{~nm}$ & 0.388605 & 5.5843 & 59.78 & $1.28499 \times 10^{7}$ \\
\hline 2 & Tox $=0.7 \mathrm{~nm}$ & 0.332582 & 2.97449 & 59.7969 & $1.58127 \times 10^{6}$ \\
\hline 3 & Tox=1nm & 0.351967 & 3.91673 & 59.80 & $2.68734 \times 10^{6}$ \\
\hline
\end{tabular}
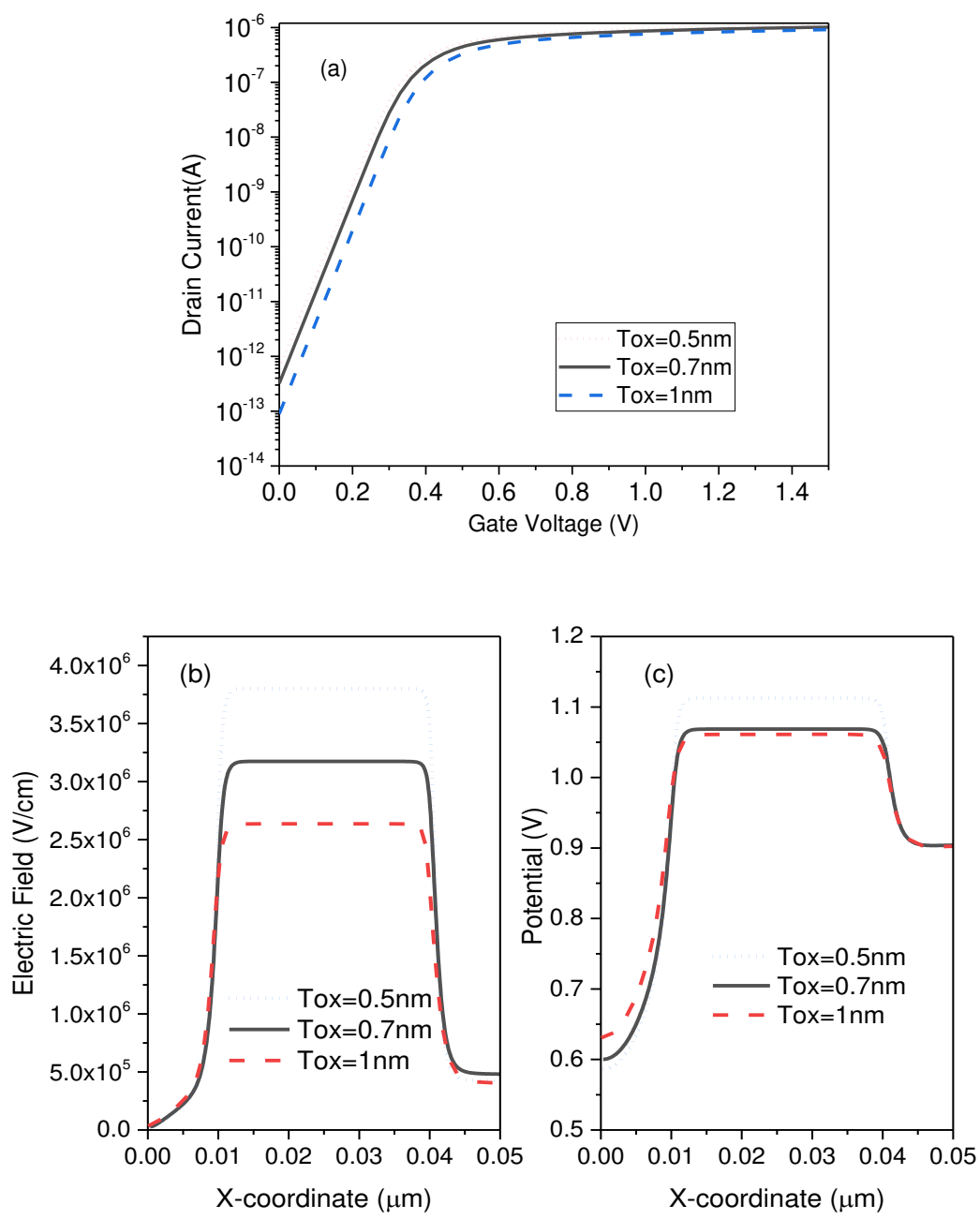

Fig. 5 Variation of the (a) $I_{D}-V_{G S}$ characteristics (b) Electric field, and (c) Surface potential of the proposed

NWFET with various $\mathrm{SiO}_{2}$ Thickness 


\section{Metal-work Function Variation}

The impact of metal work function on the various performance parameters of the proposed NWFET at room temperature is plotted and summarized in Fig. 6 and Table-IV respectively, the doping concentration of source, drain, and the channel is maintained constant as stated before. The oxide thickness of $1 \mathrm{~nm}$ is maintained constant. From the $I_{D}-V_{G S}$ characteristics graph shown in Fig. 6 (a), it is observed that changing the gate work function has enormous effects on device performance. The effect of metal work function variation on device parameters electric field and surface potential is shown in Fig. 6 (b) and Fig. 6 (c) respectively. The variations of the $\mathrm{V}_{\text {th }}$, DIBL, SS, and $\mathrm{I}_{\mathrm{ON}} / \mathrm{I}_{\mathrm{OFF}}$ ratio for various oxide thicknesses are shown in Table 4; these values are evaluated at room temperature. And it can be noticed that on increasing the metal work function both the electric field and surface potential reduce. A very low threshold voltage is observed at a low work function of $4.4 \mathrm{eV}$ but as work function increases the threshold voltage also increases. The IOFF increases with increasing gate work function. The high $\mathrm{I}_{\mathrm{ON}} / \mathrm{I}_{\mathrm{OFF}}$ ratio of order $10^{9}$ is obtained at a work function of $4.7 \mathrm{eV}$. Further increasing the gate work function reduces the energy band bending and decreases the electric field. Due to increased Ioff with further increasing work function $\mathrm{I}_{\mathrm{ON}} / \mathrm{I}_{\mathrm{OFF}}$ ratio reduces to an order of approximately $10^{3}$.

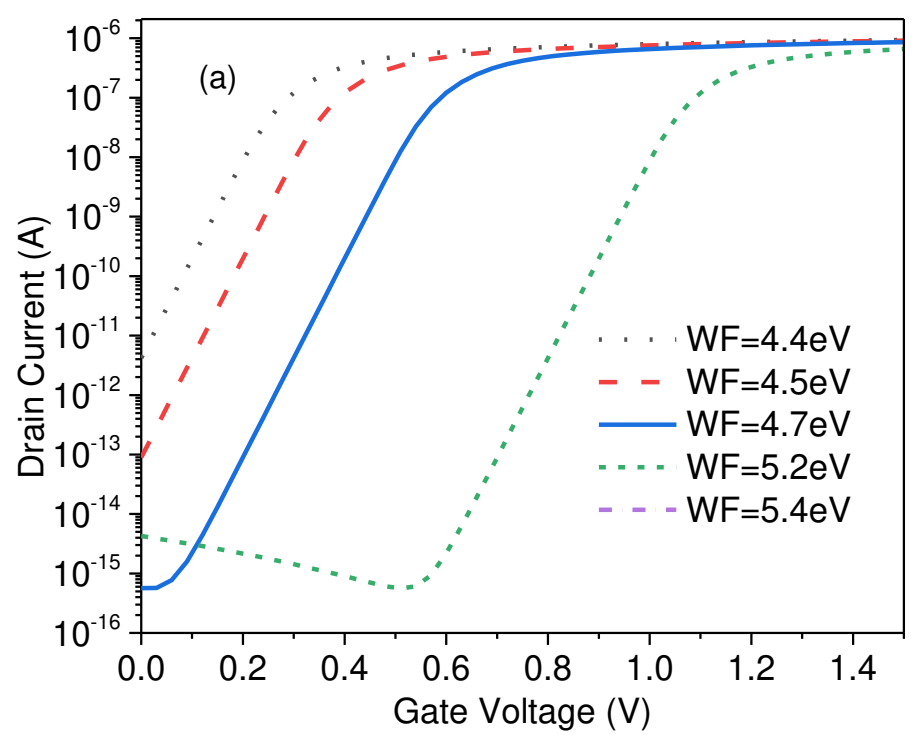



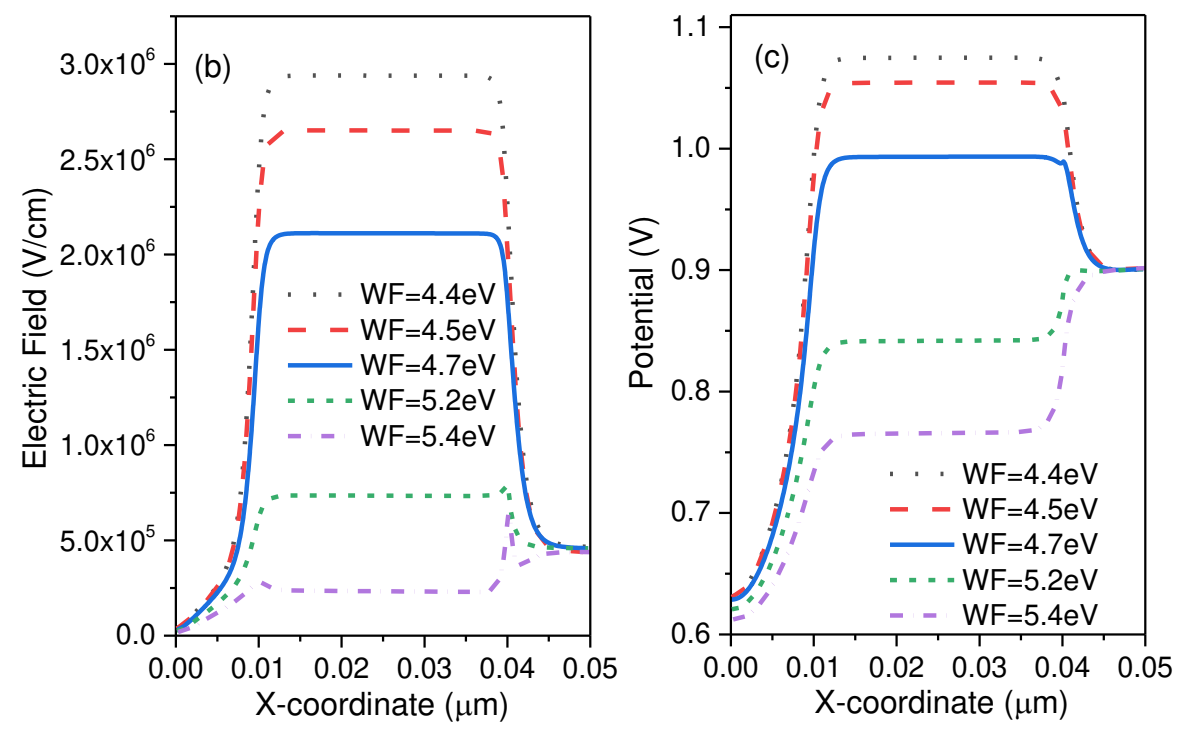

Fig. 6 Impact of the metal work function variation on the (a) $I_{D}-V_{G S}$ characteristics (b) electric field (c) and potential

Table IV: Various performance parameters for different work functions

\begin{tabular}{|l|l|l|l|l|l|}
\hline SI.No. & Work & Threshold & DIBL & Sub-threshold & Ion/loff Ratio \\
& Function(eV) & Voltage (V) & & Swing(mV/dec) & \\
\hline 1 & 4.4 & 0.289483 & 5.57479 & 59.8085 & $1.89 \times 10^{5}$ \\
\hline 2 & 4.5 & 0.351967 & 3.91673 & 59.80 & $2.68734 \times 10^{6}$ \\
\hline 3 & 4.7 & 0.589483 & 5.57474 & 59.80 & $1.16112 \times 10^{9}$ \\
\hline 4 & 5.2 & 1.08935 & -12.1039 & 59.80 & $2.3325 \times 10^{6}$ \\
\hline 5 & 5.4 & 1.2886 & -14.3178 & 59.8011 & 672.443 \\
\hline
\end{tabular}




\section{Conclusions}

This paper presents the design and performance analysis of ultra-thin cylindrical NWFET. Here, the Selfconsistent Schrodinger-Poisson model which uses the 1D NEGF methods for computation of current is invoked to model the quantum confinement effects, and then the 2-D Schrödinger equation is applied to the obtained current. Finally, the impact of temperature, oxide thickness, and metal-work function on various device performance parameters like threshold voltage, sub-threshold swing, DIBL, $\mathrm{I}_{\mathrm{ON}} / \mathrm{I}_{\mathrm{OFF}}$ ratio is evaluated along with $\mathrm{I}_{\mathrm{D}}-\mathrm{V}_{\mathrm{GS}}$ characteristics, electric field, and surface potential are investigated. The I $\mathrm{ON}_{\mathrm{N}}$ at $\mathrm{V}_{\mathrm{GS}}=1 \mathrm{~V}$ and $\mathrm{V}_{\mathrm{DS}}=1 \mathrm{~V}$ is noticed as $6.70 \times 10^{-7}(\mathrm{~A} / \mu \mathrm{m})$ whereas the $\mathrm{I}_{\mathrm{OFF}}$ is observed as $5.67 \times 10^{-16}$ $(\mathrm{A} / \mu \mathrm{m})$ and leads the $\mathrm{I}_{\mathrm{ON}} / \mathrm{I}_{\mathrm{OFF}}$ ratio in the range of $10^{9}$. A $100 \mathrm{~K}$ rise in temperature from $273 \mathrm{~K}$ to $373 \mathrm{~K}$ causes an $8 \%$ decrement in $\mathrm{V}_{\text {th, }}$ while $40 \%$ and $38 \%$ augment in DIBL and SS. The proposed design shows a high $\mathrm{I}_{\mathrm{ON}} / \mathrm{I}_{\mathrm{OFF}}$ ratio and hence will be very helpful in designing high-speed integrated circuits and ultra-thin sensor designing.

Author Contributions All authors have equally participated in the preparing of the manuscript during implementation of ideas, findings results, and writing of the manuscript.

Data Availability Current submission does not contain the pool data of the manuscript but the data used in the manuscript will be provided on request.

\section{Declarations}

Conflict of Interest The authors declare that they have no conflict of interest.

Ethical Standard The Authors accepted principles of ethical standard and they have no conflict of interest.

Consent to Participate Informed consent.

Consent for Publication Consent is granted. 


\section{Acknowledgements and Funding Not Applicable}

\section{References}

[1] Hoefflinger, B. ed., 2012. Chips 2020: a guide to the future of nanoelectronics. Springer Science \& Business Media.

[2] Cheung, K.P., 2010, April. On the $60 \mathrm{mV} / \mathrm{dec} @ 300 \mathrm{~K}$ limit for MOSFET subthreshold swing. In Proceedings of 2010 International Symposium on VLSI Technology, System and Application (pp. 72-73). IEEE

[3] Ansari, M.T., Husain, M.M. and Rafat, M., 2018, November. Modeling of carbon chain device employing quantum mechanical method: a hybrid diode. In 2018 IEEE Electron Devices Kolkata Conference (EDKCON) (pp. 1-7). IEEE.

[4] Sahay, S. and Kumar, M.J., 2017. Diameter dependence of leakage current in nanowire junctionless field effect transistors. IEEE Transactions on Electron Devices, 64(3), pp.1330-1335.

[5] Tamersit, K., 2020. Improved performance of nanoscale junctionless carbon nanotube tunneling FETs using dual-material source gate design: A quantum simulation study. AEU-International Journal of Electronics and Communications, 127, p.153491.

[6] Frank, D.J., Dennard, R.H., Nowak, E., Solomon, P.M., Taur, Y. and Wong, H.S.P., 2001. Device scaling limits of Si MOSFETs and their application dependencies. Proceedings of the IEEE, 89(3), pp.259-288.

[7] Wadhwa, G. and Singh, J., 2020. Implementation of linearly modulated work function A $\sigma$ B $1-\sigma$ gate electrode and $\mathrm{Si} 0.55 \mathrm{Ge} 0.45 \mathrm{~N}+$ pocket doping for performance improvement in gate stack vertical-TFET. Applied Physics A, 126(11), pp.1-11.

[8] Moore, G.E.,Cramming more components onto integrated circuits, Electronics, Volume 38, Number 8, April 19, 1965.

[9] Bayani, A.H., Voves, J. and Dideban, D., 2018. Effective mass approximation versus full atomistic model to calculate the output characteristics of a gate-all-around germanium nanowire field effect transistor (GAA-GeNW-FET). Superlattices and Microstructures, 113, pp.769-776

[10] Kumar, S. and Raj, B., 2015. Compact channel potential analytical modeling of DG-TFET based on Evanescent-mode approach. Journal of Computational Electronics, 14(3), pp.820-827. 
[11] Gupta, A.K., Raman, A. and Kumar, N., 2019. Design and investigation of a novel charge plasma-based core-shell ring-TFET: analog and linearity analysis. IEEE Transactions on Electron Devices, 66(8), pp.3506-3512.

[12] Kumar, N., Mushtaq, U., Amin, S.I. and Anand, S., 2019. Design and performance analysis of dual-gate all around core-shell nanotube TFET. Superlattices and Microstructures, 125, pp.356-364.

[13] Kumar, N. and Raman, A., 2019. Design and investigation of charge-plasma-based work function engineered dual-metal-heterogeneous gate $\mathrm{Si}-\mathrm{Si}$ 0.55 Ge 0.45 GAA-cylindrical NWTFET for ambipolar analysis. IEEE Transactions on Electron Devices, 66(3), pp.1468-1474.

[14] Singh, A., Khosla, M. and Raj, B., 2017. Design and analysis of electrostatic doped Schottky barrier CNTFET based low power SRAM. AEU-International Journal of Electronics and Communications, 80, pp.67-72.

[15] Appenzeller, J., Lin, Y.M., Knoch, J. and Avouris, P., 2004. Band-to-band tunneling in carbon nanotube field-effect transistors. Physical review letters, 93(19), p.196805.

[16] Lundstrom, M. and Ren, Z., 2002. Essential physics of carrier transport in nanoscale MOSFETs. IEEE Transactions on Electron Devices, 49(1), pp.133-141. 